\section{Estimating Retinal Blood Flow Velocities by Optical Coherence Tomography}

Gerald Seidel, MD; Gerold Aschinger, MSc; Christoph Singer; Sereina Annik Herzog, PhD; Martin Weger, MD; Anton Haas, MD; René Marcel Werkmeister, PhD; Leopold Schmetterer, PhD; Gerhard Garhöfer, MD

IMPORTANCE While optical coherence tomography (OCT) angiography has been considered to evaluate retinal capillary blood flow instead of fluorescein angiography, the reflectance pattern of blood vessels on structural OCT might also provide retinal capillary flow data in the absence of fluorescein angiography. This potential has been insufficiently explored, despite promising data concerning a possible relationship between the reflectance pattern of blood vessels and their perfusion velocity in a laboratory setting.

OBJECTIVE To evaluate the potential of retinal blood flow velocity estimation by structural OCT.

DESIGN, SETTING, AND PARTICIPANTS Cross-sectional observational study conducted from June to November 2015 at a tertiary clinical referral center. Sixty arteries (the superior and inferior temporal arteries) from 30 eyes of 30 patients (17 female, 13 male) were included in the study.

MAIN OUTCOMES AND MEASURES Based on the intraluminal contrast patterns of retinal arteries on OCT, 3 independent graders categorized the blood flow velocities as low, medium, or high. These results and the results from a software-based intraluminal contrast analysis were compared with the retinal blood flow velocities measured by video fluorescein angiography.

RESULTS Among the 30 eyes of 30 patients (mean [SD] age, 72.6 [12.3] years; 17 female, 13 male), 15 were controls without retinal occlusion, 6 had a branch retinal artery occlusion, and 9 had a central retinal artery occlusion. When discriminating between low flow velocities and medium or high flow velocities, the graders' sensitivity ranged from $88.2 \%$ to $100 \%$ (grader 1: $88.2 \%$; $95 \% \mathrm{Cl}$, 63.6\%-98.5\%; grader 2: $88.2 \%$; $95 \% \mathrm{Cl}, 63.6 \%-98.5 \%$; and grader 3: $100 \% ; 95 \% \mathrm{Cl}, 69.8 \%-100 \%$ ) and their specificity ranged from $97.6 \%$ to $100 \%$ (grader 1 : $100 \%$; $95 \% \mathrm{Cl}, 87.7 \%-100 \%$; grader 2: $97.6 \%$; $95 \% \mathrm{Cl}, 87.4 \%-99.9 \%$; and grader 3: $100 \%$; $95 \% \mathrm{Cl}, 87.7 \%-100 \%)$. The $\mathrm{k}$ coefficients of the comparison between the 3 graders and the angiography were $0.77(95 \% \mathrm{Cl}, 0.60-0.93 ; P<.001), 0.64(95 \% \mathrm{Cl}, 0.44-0.83 ; P<.001)$, and $0.87(95 \% \mathrm{Cl}, 0.74-0.99 ; P<.001)$. In the computer-based assessment, the contrast reduction of the intraluminal pattern could be numerically expressed in a specific coefficient in the model $\left(I_{2}\right.$, describing the angular change of the backscattering intensity in the model), which presented nonoverlapping intervals between low flow velocities and medium or high flow velocities (mean [SD] $I_{2}, 0.3$ [5.3], 20.4 [6.4], and 21.7 [4.0], respectively).

CONCLUSIONS AND RELEVANCE This study suggests that a low retinal blood flow velocity reflects in a visually distinct contrast reduction of the intraluminal pattern of retinal vessels on OCT. Larger studies are required to assess the clinical benefits.

JAMA Ophthalmol. 2016;134(10):1104-1110. doi:10.1001/jamaophthalmol.2016.2507 Published online August 4, 2016.
Author Affiliations: Department of Ophthalmology, Medical University of Graz, Graz, Austria (Seidel, Singer, Weger, Haas); Center for Medical Physics and Biomedical Engineering, Medical University of Vienna, Vienna Austria (Aschinger, Werkmeister, Schmetterer); Institute for Medical Informatics, Statistics and Documentation, Medical University of Graz, Graz, Austria (Herzog); Department of Clinical Pharmacology, Medical University of Vienna, Vienna, Austria (Schmetterer, Garhöfer).

Corresponding Author: Gerald Seidel, MD, Department of Ophthalmology, Medical University of Graz, Auenbruggerplatz 4, 8036 Graz, Austria (gerald.seidel @medunigraz.at). 
S ince 1991, optical coherence tomography (OCT) sweepingly spread throughout ophthalmology and thereby revolutionized noninvasive imaging of ocular structures. However, a major limitation of structural OCT is that it apparently provides no direct functional information, such as perfusion. This issue is addressed by OCT angiography and Doppler OCT. ${ }^{1,2}$ Devices for OCT angiography are not yet widely adopted, and devices for Doppler OCT are not even commercially available. Thus, today in many practices, fluorescein angiography is used for the imaging of retinal perfusion.

Compared with fluorescein angiography, OCT is faster, easier to perform, and less unpleasant to the patient and, most important, has a better safety profile because it is noninvasive. These advantages warrant a closer look at the diagnostic potential of structural OCT images. While functional information cannot be assessed directly from a frozen image, some information, blood flow velocity in particular, can be assessed indirectly. For this purpose, the intraluminal backscattering pattern on OCT cross sections from retinal vessels can serve as a marker for the blood flow velocity. ${ }^{3}$

In such scans, an hourglass-shaped pattern arises from the erythrocyte's orientation within the vessel's lumen. When erythrocytes travel in a tubelike structure, they align themselves with their disk plane parallel to the vessel wall. Thus, when these erythrocytes are scanned by the light beam of OCT, they are oriented perpendicular on the top and bottom of the vessel, parallel on the sides, and relatively loose in the center (Figure 1). Because most reflection occurs when the beam meets a perpendicular interface, healthy retinal vessels exhibit higher backscattering on their top and bottom than on their sides and in their center. This generates the hourglasslike appearance. The faster the blood flows, the tidier the erythrocytes align, which in turn leads to a more pronounced demarcation between high- and low-reflecting areas. A slower blood flow, on the other hand, correlates with a lower contrast or even absence of this pattern (Figure 2). This has been shown experimentally in glass tubes and in the saphenous artery of mice. ${ }^{3}$ The presence of this pattern has been confirmed in a clinical setting by Willerslev et al, ${ }^{4-6}$ who also indicated a relationship between an altered intraluminal reflectivity and altered blood flow circumstances such as vessel branching, emboli, or ocular ischemic syndrome.

The aim of this study was to assess the potential of the hourglass-shaped intravascular pattern on OCT to serve as a tool for estimating the retinal blood flow velocity in humans.

\section{Methods}

\section{Data Collection}

After a planning phase in January 2015, the study was conducted at a tertiary referral center from June to November 2015. Patients with a branch retinal artery occlusion, with a central retinal artery occlusion, and without a retinal artery occlusion were included. The latter group comprised patients who underwent fluorescein angiography for abnormalities in the fellow eye such as retinal vein occlusions or age-related macular degeneration. All patients underwent an ophthalmologic

\section{Key Points}

Question Can retinal blood flow velocity be estimated from structural optical coherence tomographic scans?

Findings In this cross-sectional study of 30 eyes from 30 patients, retinal blood flow velocity could be visually graded as low, medium, or high flow velocity via assessment of the intraluminal backscattering pattern on optical coherence tomography by 3 independent graders. When discriminating low flow velocities from medium or high flow velocities, sensitivity ranged from $88.2 \%$ to $100 \%$ and specificity ranged from $97.6 \%$ to $100 \%$.

Meaning These data suggest that a low retinal blood flow velocity reflects in a visually distinct contrast reduction of the intraluminal pattern of retinal vessels on optical coherence tomography.

Figure 1. Schematic of Moving Erythrocyte's Alignment in Relation to the Optical Coherence Tomographic Scan Beam

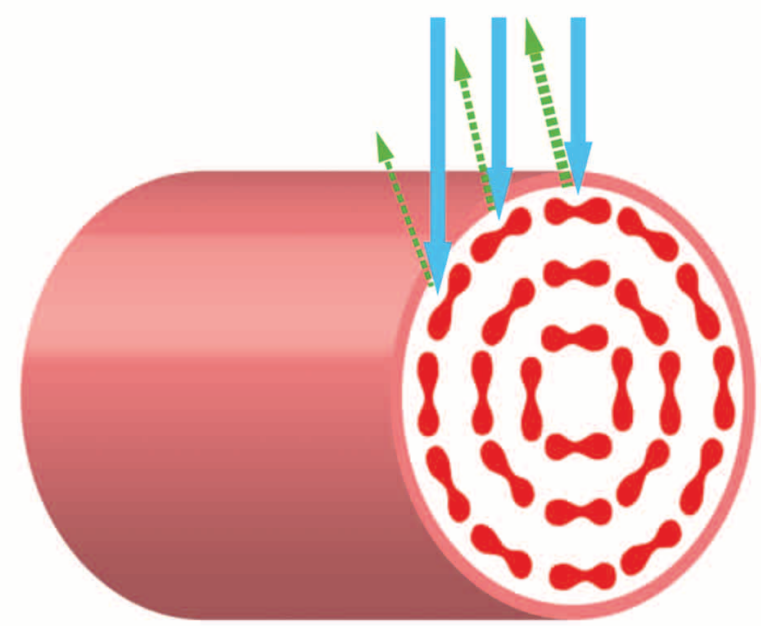

Blue arrows indicate the optical coherence tomographic scan beam; green arrows, backscattered light. The reflection is higher for perpendicular erythrocytes than for parallel erythrocytes. Adapted from Cimalla et al. ${ }^{3}$

examination including medical history, best-corrected visual acuity assessment, intraocular pressure measurement (Goldmann tonometer AT 900; Haag-Streit AG), slitlamp examination, indirect ophthalmoscopy, fundus photography (FF450 fundus camera; Carl Zeiss Meditec), optical biometry (IOLMaster; Carl Zeiss Meditec), OCT (Spectralis OCT; Heidelberg Engineering), and video fluorescein angiography (Heidelberg Retina Angiograph 2; Heidelberg Engineering). Prior to the study initiation, the institutional review board of the Medical University of Graz approved all study procedures, the study protocol, and the informed consent form. The latter was signed by all study participants in case of agreement to participate in the study after a personal colloquium with the principal investigator (G.S.). All participants were recruited from patients presenting at the study hospital. The study adhered to the Declaration of Helsinki. ${ }^{7}$ 
Figure 2. Cross Sections of Retinal Arteries at Varying Blood Flow Velocities on Optical Coherence Tomography

A Low flow velocity

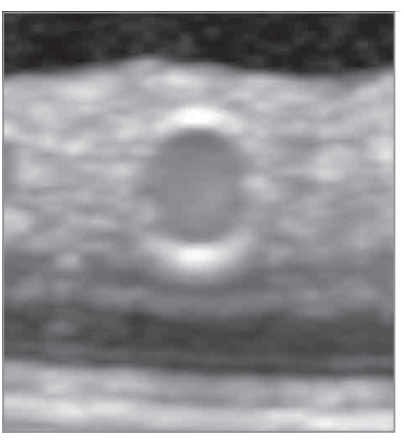

B Medium flow velocity

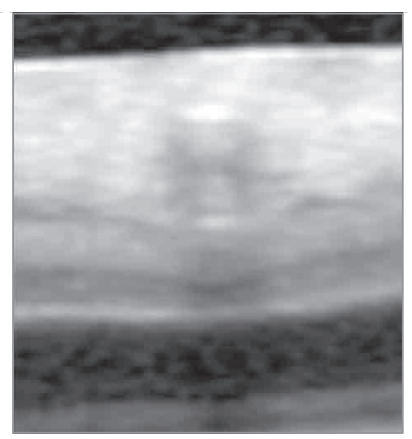

D Erythrocyte alignment

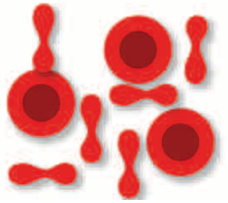

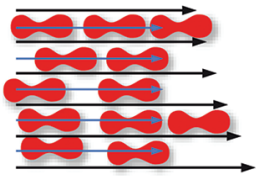

C High flow velocity

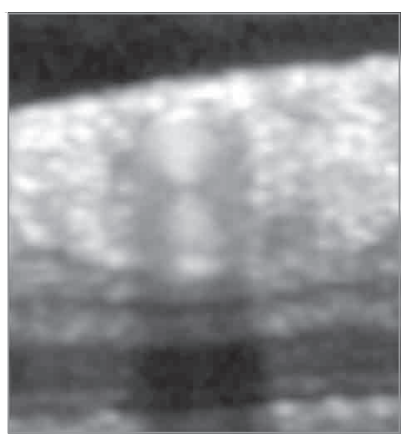

The contrast of the intraluminal pattern on optical coherence tomography increased from low flow velocity $(<1.5 \mathrm{~mm} / \mathrm{s})(\mathrm{A})$, over medium flow velocity $(1.5-3.0 \mathrm{~mm} / \mathrm{s})$ (B), to high flow velocity $(>3.0 \mathrm{~mm} / \mathrm{s})$ (C). D, The responsible erythrocyte alignment is schematized under each optical coherence tomographic image (adapted from Cimalla et $\mathrm{al}^{3}$ ). Black arrows indicate flow velocity; blue arrows, shear stress.

\section{OCT Image Acquisition}

We used Spectralis OCT with version 6.0.9 software (Heidelberg Engineering) to record $20^{\circ}$ single line scans perpendicular to the inferior and superior temporal retinal arteries. The scan's distance to the optic disc measured approximately 1 disc diameter. Each of these vessel cross sections comprised 100 averaged images (100 frames) in the high-resolution mode of the device. To assess a potential influence of a varying amount of averaging, a subgroup of patients was additionally imaged with 50 frames. For all recordings, the built-in eye tracking software ensured exact position correspondence.

\section{Fluorescein Angiography}

Within 30 minutes after OCT was performed, video fluorescein angiography was performed with a confocal scanning laser system (Heidelberg Retina Angiograph 2, version 5.7.4 software; Heidelberg Engineering). The procedure has been described in detail elsewhere. ${ }^{8-11}$ In short, each patient received a 5-mL bolus of $10 \%$ fluorescein dye into the antecubital vein and a video of the posterior pole was digitally recorded with a confocal laser ophthalmoscope with a $50^{\circ}$ objective. On the resulting images, we selected the temporal major retinal branch arteries and measured the distance from a location close to the optic rim to a location on the same artery as distant as possible without major arterial branching in between, using the device's built-in software. The time the fluorescein took to travel this distance was noted and from the resulting 2 parameters the fluorescein's traveling velocity was calculated as distance over time. This served as an estimate for the true blood flow velocity and was categorized as low flow velocity $(<1.5 \mathrm{~mm} / \mathrm{s})$, medium flow velocity $(1.5-3.0 \mathrm{~mm} / \mathrm{s})$, or high flow velocity $(>3.0 \mathrm{~mm} / \mathrm{s})$.

\section{Semiautomated Image Assessment}

For software analysis, a self-developed semiautomated approach was implemented in which the user first had to mark the vessel lumen in the OCT B-scan. Then, the software analysis of the intraluminal intensity pattern was carried out based on the method proposed by Cimalla et al. ${ }^{3}$ For this method, the following function is fitted into the intensity variation along a circular path at half the vessel radius:

$I(\varphi)=I_{\mathrm{O}}+I_{1} \cdot \cos (\varphi)+I_{2} \cdot \cos (2 \varphi)+I_{3} \cdot \cos (3 \varphi)+I_{4} \cdot \cos (4 \varphi)$,

where $\varphi$ is the angle of rotation with respect to the direction of the probing beam, $I_{\mathrm{O}}$ is the background intensity, $I_{1}$ is the OCT signal intensity loss over the vessel depth, $I_{2}$ and $I_{4}$ characterize the angular variation in red blood cell backscattering along the circular path, and $I_{3}$ represents the nonlinear intensity variations along the vessel depth.

The parameter $I_{2}$ best describes the hourglass-shaped intensity patterns seen in the vessel cross sections and is therefore the most promising indicator for flow velocity. Figure 3 shows an exemplary reflection analysis and the resulting model fit.

\section{Grader-Based Image Assessment}

To evaluate a retinal specialist's ability to visually discriminate contrast changes of the intraluminal pattern on OCT, the vessel cross sections were presented to 3 retina specialists (A.H., M.W., and G.S.) who were informed about the concept of the hourglass sign but masked to the diagnosis, to the belonging of any vessel to a particular eye, and to the blood flow velocities of the individual vessels. Each grader evaluated all vessels in a single session independent from the other graders. To preclude an influence by the infrared fundus image of the OCT, a technician loaded each scan and subsequently 


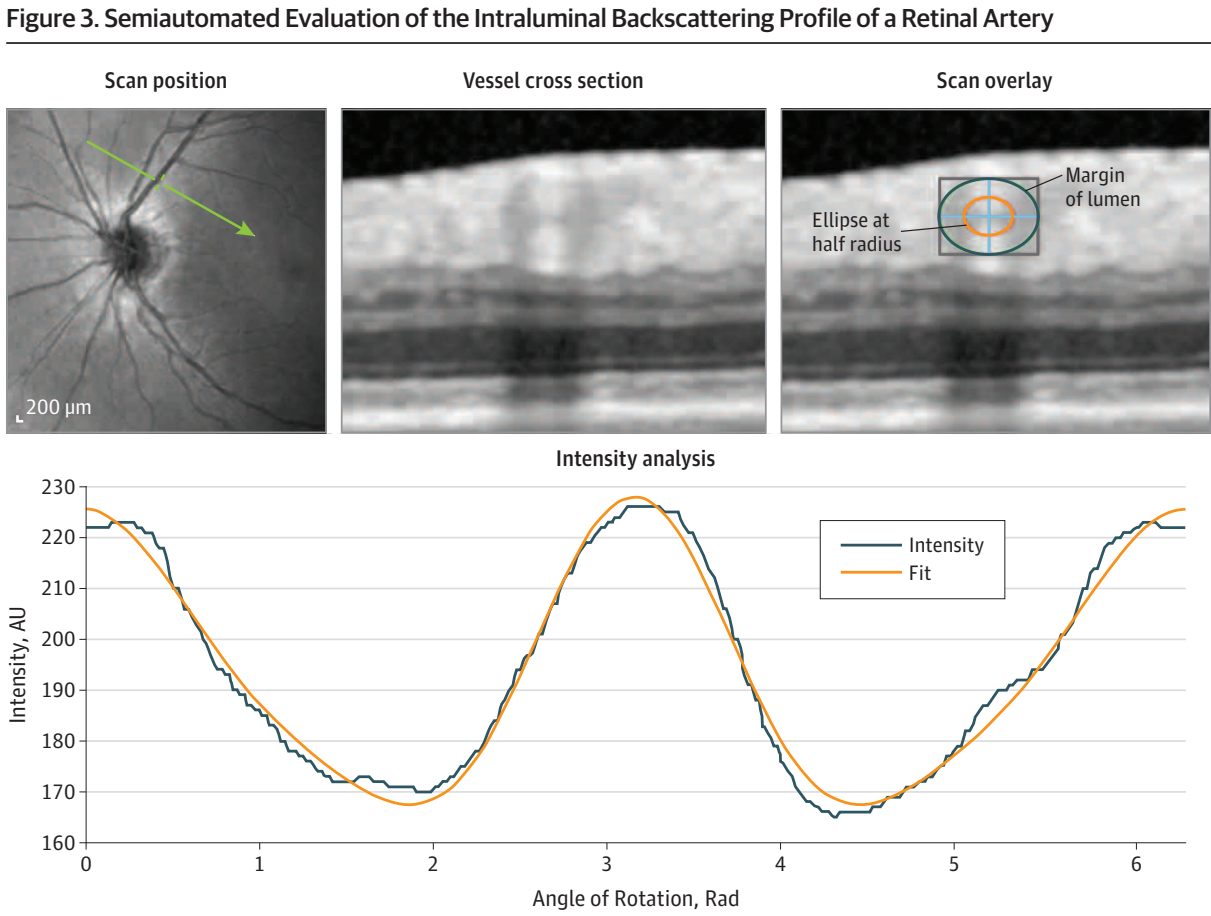

Each optical coherence tomographic scan was positioned perpendicular to the temporal retinal artery. Arrow indicates the position of the scan beam. The backscattering intensity was measured at half the vessel diameter. In vessels with high flow velocity, this resulted in bimodal intensity curve modeling. $\mathrm{AU}$ indicates arbitrary units. covered the infrared image on the monitor. The grader could then adjust the OCT image to his preferred magnification and scaling on the device and grade each vessel analogous to the angiography categories as low flow velocity, medium flow velocity, or high flow velocity by his judgment of the intraluminal reflection pattern (Figure 2).

\section{Statistical Analysis}

We analyzed the extent of agreement for the categories low flow velocity, medium flow velocity, and high flow velocity between each grader and the angiography by estimating Cohen $\kappa$ statistics. We estimated the sensitivity and specificity of the graders to identify low flow velocity vs medium or high flow velocity using the angiography categories as gold standard. Results are presented with $95 \%$ confidence intervals. Because this study was considered a pilot study, no sample size calculation was performed. Taking the number of patients into account, this is an exploratory analysis. Data are shown as mean (standard deviation) or median (interquartile range [IQR]). All analyses were performed using R version 3.2.2 statistical software (R Foundation).

\section{Results}

Vessels

We included 60 arteries (the superior and inferior temporal arteries) from 30 eyes of 30 patients ( 17 female, 13 male) with a mean (SD) age of 72.6 (12.3) years. Of the 30 eyes, 15 were controls without a retinal artery occlusion, 6 had a branch retinal artery occlusion, and 9 had a central retinal artery occlusion. The median time from the reported symptom onset to the study procedures was 1.9 days (range, 5 hours to 63 days; mean [SD], 6.3 [13.9] days).
Based on the angiographic flow analysis, 17 arteries (28.3\%) showed low flow velocity, 6 (10.0\%) showed medium flow velocity, and 37 (61.7\%) showed high flow velocity. The median flow velocities for the 3 groups were $0.1 \mathrm{~mm} / \mathrm{s}$ (IQR, 0.05-0.3 $\mathrm{mm} / \mathrm{s}$ ), $2.4 \mathrm{~mm} / \mathrm{s}$ (IQR, 2.3-2.5 mm/s), and $6.7 \mathrm{~mm} / \mathrm{s}$ (IQR, 4.9$8.4 \mathrm{~mm} / \mathrm{s})$, respectively.

\section{OCT Grader Evaluation}

A single OCT cross section of a healthy vessel was excluded owing to poor image quality, leaving 59 vessels to compare with the angiographic findings. The graders could discriminate a vessel with low flow velocity from a vessel with medium or high flow velocity with a sensitivity of $88.2 \%$ to $100 \%$ (grader 1: $88.2 \%$; $95 \%$ CI, 63.6\%-98.5\%; grader 2: 88.2\%; 95\% CI, 63.6\%-98.5\%; and grader 3: 100\%; 95\% CI, 69.8\%-100\%) and a specificity of $97.6 \%$ to $100 \%$ (grader 1: 100\%; 95\% CI, $87.7 \%-100 \%$; grader 2: $97.6 \%$; 95\% CI, 87.4\%-99.9\%; and grader 3: 100\%; 95\% CI, 87.7\%-100\%).

The $к$ coefficients of the comparison between the 3 graders and the angiography were 0.77 (95\% CI, 0.60-0.93; $P<.001), 0.64$ (95\% CI, 0.44-0.83; $P<.001)$, and 0.87 (95\% CI, 0.74-0.99; $P<.001$ ), ie, the agreement between the graders' estimates and the angiography measurements regarding the 3 blood flow categories was good to very good.

There was no difference if the graders rated a 50-frame or 100-frame image. The subset in which both frame rates were available included 49 vessels, and the $\kappa$ coefficients of the graders when comparing their 50-frame choices with their 100frame choices ranged from 0.86 to 0.96 (grader 1: $0.87 ; 95 \%$ CI, 0.72-1.01; $P<.001$; grader 2: 0.86; 95\% CI, 0.71-1.01, $P<.001$; and grader 3: 0.96; 95\% CI, 0.87-1.04; $P<.001$ ), ie, the agreement between the 2 frame rates was very good. The agreement with the angiographic findings was slightly poorer with 50 frames than 100 frames in 2 graders but slightly 

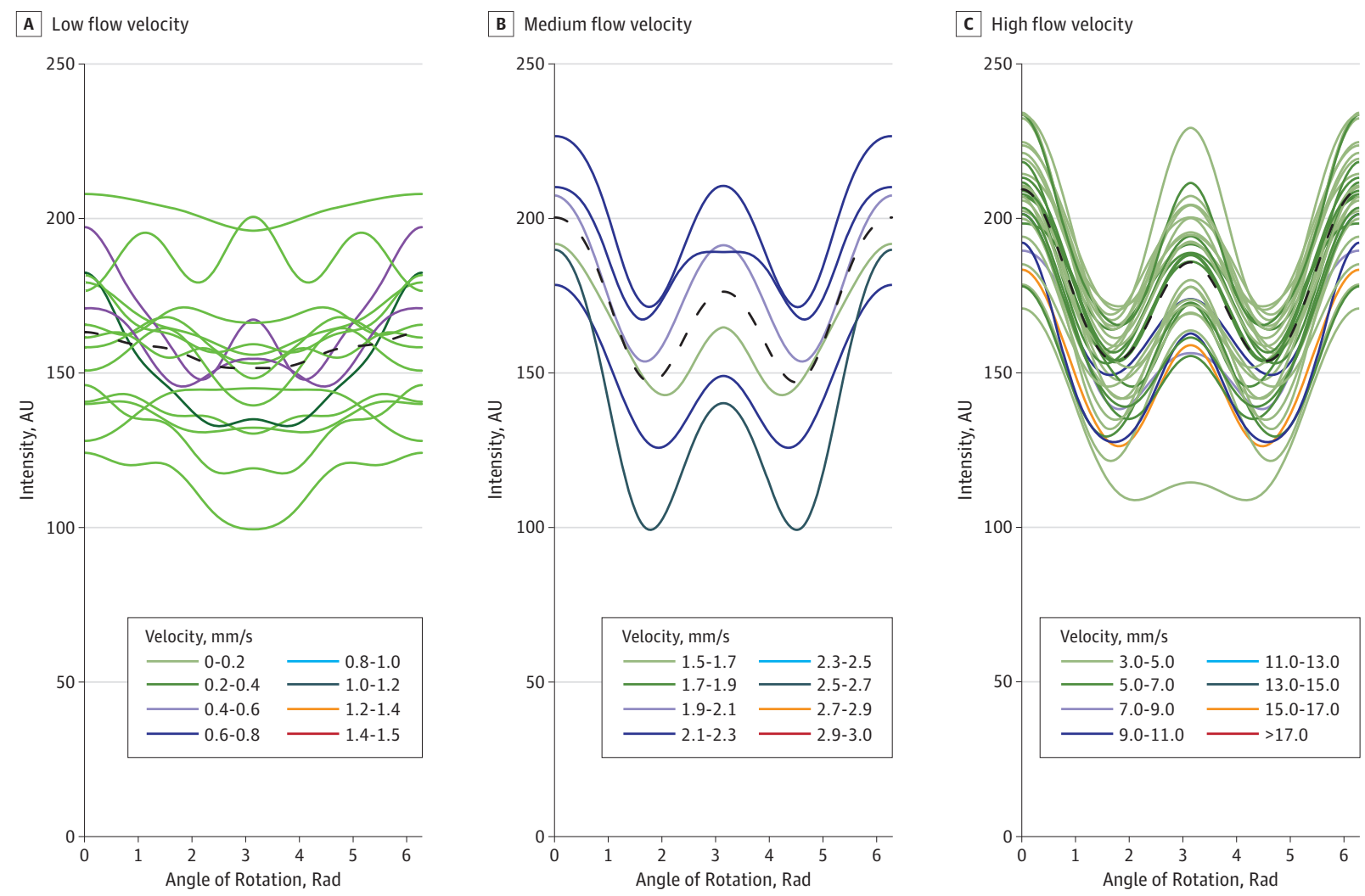

While the profiles in the vessels with low flow velocity were irregular $(A)$, those with medium (B) and high (C) flow velocity exhibited a characteristic bimodal

velocities within a single category. Dashed lines indicate mean backscattering profile. This pattern also seemed more pronounced in vessels with higher intensity for each category; $\mathrm{AU}$, arbitrary units.

better with 50 frames than 100 frames in 1 grader. In any case, the agreement remained high (grader $1: \mathrm{K}=0.69 ; 95 \% \mathrm{CI}, 0.48$ $0.90 ; P<.001$; grader 2 : $\mathrm{K}=0.77 ; 95 \% \mathrm{CI}, 0.57-0.96 ; P<.001$; and grader 3: $\mathrm{K}=0.86 ; 95 \% \mathrm{CI}, 0.71-1.00 ; P<.001)$.

\section{Semiautomated OCT Evaluation}

The mean (SD) $I_{2}$, which describes the angular change of the backscattering intensity in the model, was 0.3 (5.3) in the low flow velocity group, 20.4 (6.4) in the medium flow velocity group, and 21.7 (4.0) in the high flow velocity group. Notably, there was no overlap of the values of the low flow velocity group with the values of the medium or high flow velocity groups. With increasing flow velocity, the model resulted in an increasingly regular bimodal fit. Figure 4 shows the backscattering intensity profiles of all vessels according to their categorization on angiography.

In the high flow velocity group, 1 vessel fell out of line, lacking the middle hump and having the lowest $I_{2}, 13.4$, as compared with the other vessels in this category. On review, this vessel was measured at a bend adjacent to the corresponding vein (Figure 5). On the grader-based evaluation of this vessel, both graders 2 and 3 incorrectly estimated a medium flow velocity, while grader 1 categorized it correctly as having a high flow velocity. This vessel remained in the statistical analysis.

\section{Discussion}

In this prospective, single-center case series, we evaluated the possibility of estimating retinal blood flow velocity on structural OCT B-scans. Our data show that the intraluminal backscattering profile on such a scan enables the visual estimation of low blood flow velocities in retinal vessels by human graders. The results of a semiautomated approach are promising but should be validated prospectively.

Previously proposed techniques for evaluating retinal blood flow velocity include fluorescein angiography, ${ }^{12}$ laser Doppler velocimetry, ${ }^{13}$ color Doppler imaging, ${ }^{14}$ the laser speckle method, ${ }^{15}$ motion-contrast velocimetry, ${ }^{16}$ Doppler OCT, ${ }^{1}$ and optical angiography. ${ }^{17}$ While most of these methods focus on a more quantitative assessment, compared with them the blood flow velocity estimation with traditional spectral-domain OCT has its advantages. First, owing to its noninvasive nature and acquisition speed, it is easier and safer to obtain in routine practice than most of the other methods. Second, it is relatively cheap, as most ophthalmic clinics already use spectral-domain OCT. Third, it can be easily repeated within short periods. For example, fluorescein angiography takes 24 hours after initial use until naive imaging can be obtained. 


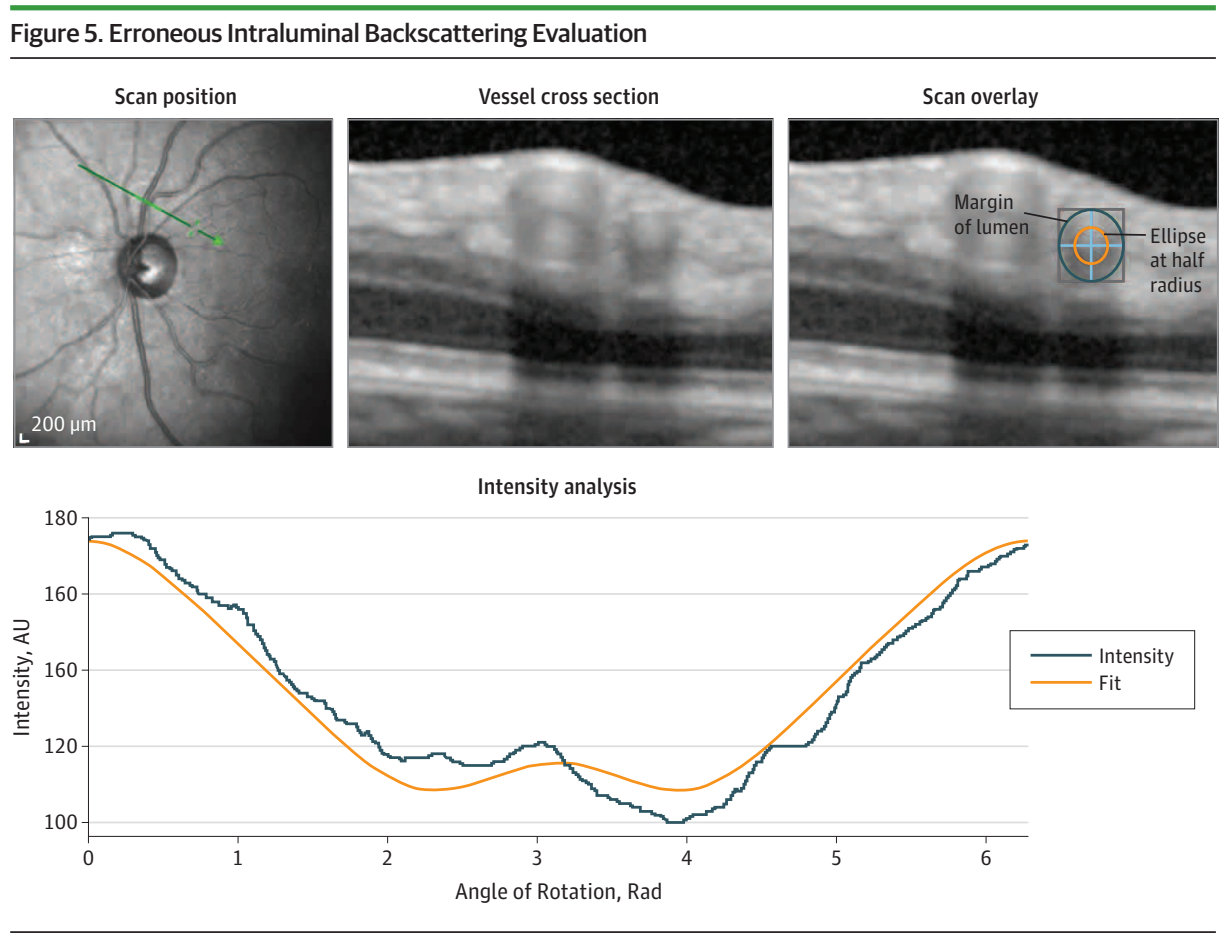

In 1 artery with high blood flow velocity, the scan position (arrow) at a bend close to a retinal vein resulted in low contrast in the inferior half of the vessel and a lacking middle hump on the intensity profile. AU indicates arbitrary units.
Our data show that a lower flow velocity correlated with reduced contrast of the OCT pattern, and an absent pattern as detected by visual grading highly correlated with a severely diminished blood flow velocity. This was reflected in our study population by a positive predictive value of 0.93 and a negative predictive value of 0.91 for all graders combined. Of all gradings with 100 frames, only once was a vessel incorrectly graded as low flow velocity (false-positive) and 4 times was a vessel of the low flow velocity group missed (false-negative).

On the semiautomated profile, a faster blood flow translated into a more pronounced double hump pattern. This could be numerically expressed in a specific coefficient in the model $\left(I_{2}\right)$, which presented nonoverlapping intervals between low flow velocities and medium or high flow velocities. A slower flow was associated with an irregular shape of the backscattering profile, which contrasted with an increasing regularity of a double hump at faster velocities. This was true not only between the flow categories but also within the categories themselves. In other words, the flow velocity values at the higher end of each category's spectrum tended to show a more pronounced double hump than those at the lower end of each category.

We did not directly compare the grader-based and semiautomated assessments because no estimates for any of the coefficients had been available for humans. The groundwork by Cimalla et $\mathrm{l}^{3}$ dealt with an in vitro model and the saphenous artery of mice, and extrapolation to the human eye did not seem adequate. However, the values for $I_{2}$ in the present study could serve as a reference point for future investigations.

The following limitations regarding the presented data need to be considered. First, any categorization of retinal blood flow velocity is arbitrary to some degree. Even in healthy individuals, the retinal blood flow velocity varies greatly and the coefficients of variation of repeated measures for video fluorescein angiography range from $10 \%$ to $25 \% .{ }^{9}$ The reported velocities between different methods vary to an even greater extent, probably not least because they measure different aspects of blood movement such as erythrocyte velocity or plasma velocity. ${ }^{9,16,18,19}$ Moreover, the blood flow velocity is highly dependent on the vessel diameter. ${ }^{20,21}$

We based our categorization on the values for fluorescein traveling velocities by Wolf et al, ${ }^{9}$ who reported a mean (SD) velocity of $6.4(1.7) \mathrm{mm} / \mathrm{s}$ in temporal retinal arteries of healthy adults. This means that, given a normal distribution, fewer than $1 \%$ of healthy temporal retinal arteries should have blood flow velocities slower than $1.5 \mathrm{~mm} / \mathrm{s}$. As our values for the healthy vessels match those previously reported, the cutoff values used in the present study seem to be a reasonable estimate.

Second, while the measurement with OCT is fairly robust, improper positioning of the scan can produce misleading results. This was the case in 1 vessel measurement at a bend that led to poor contrast of the intraluminal pattern and a tendency to underestimate the flow velocity. The reason for the altered contrast might lie in the loss of the parabolic shape of the blood flow velocity profile. ${ }^{22}$ A fairly straight segment would be the preferred location to measure.

Third, this study provides limited data to conclude whether the discrimination of varying blood flow velocities can be translated to the intermediate range of blood flow variations. However, our results demonstrated no difference in the coefficient between our medium and high velocity groups (mean [SD] $I_{2}, 20.4$ [6.4] vs 21.7 [4.0], respectively).

Fourth, we cannot fully exclude that other OCT signs that indicate retinal ischemia such as retinal nerve fiber layer swelling or increased hyperreflectivity of the inner portion of the outer plexiform layer may have influenced the graders. ${ }^{23}$ 
However, 7 OCT images showed pronounced retinal nerve fiber layer swelling and a prominent inner limiting membrane sign while having medium or high blood flow velocities, and 1 image of a retinal vessel with a low blood flow velocity lacked retinal nerve fiber layer swelling and a prominent inner limiting membrane sign. In all these circumstances, the graders differentiated the vessels with low flow velocities from those with medium or high flow velocities correctly. This emphasizes that the intraluminal backscattering profile is less a tool for diagnosing retinal artery occlusions and more a tool for judging the blood flow velocity at the time of measurement.

\section{Conclusions}

A low retinal blood flow velocity is reflected in a visually distinct contrast reduction of the intraluminal pattern of retinal vessels on OCT B-scans.

\section{ARTICLE INFORMATION}

Accepted for Publication: June 5, 2016.

Published Online: August 4, 2016. doi:10.1001/jamaophthalmol.2016.2507.

Author Contributions: Dr Seidel had full access to all of the data in the study and takes responsibility for the integrity of the data and the accuracy of the data analysis.

Study concept and design: Seidel, Aschinger, Schmetterer, Garhöfer.

Acquisition, analysis, or interpretation of data: All authors.

Drafting of the manuscript: Seidel.

Critical revision of the manuscript for important intellectual content: All authors.

Statistical analysis: Seidel, Herzog.

Administrative, technical, or material support

Seidel, Singer, Weger, Haas, Werkmeister,

Schmetterer.

Study supervision: Seidel, Werkmeister, Garhöfer.

Conflict of Interest Disclosures: All authors have completed and submitted the ICMJE Form for Disclosure of Potential Conflicts of Interest and none were reported.

\section{REFERENCES}

1. Leitgeb RA, Werkmeister RM, Blatter C, Schmetterer L. Doppler optical coherence tomography. Prog Retin Eye Res. 2014;41:26-43.

2. Keane PA, Sadda SR. Retinal imaging in the twenty-first century: state of the art and future directions. Ophthalmology. 2014;121(12):2489-2500.

3. Cimalla P, Walther J, Mittasch M, Koch E. Shear flow-induced optical inhomogeneity of blood assessed in vivo and in vitro by spectral domain optical coherence tomography in the $1.3 \mu \mathrm{m}$ wavelength range. J Biomed Opt. 2011;16(11):116020.

4. Willerslev A, Li XQ, Cordtz P, Munch IC, Larsen M. Retinal and choroidal intravascular spectral-domain optical coherence tomography. Acta Ophthalmol. 2014:92(2):126-132.
5. Willerslev A, Li XQ, Munch IC, Larsen M. Flow patterns on spectral-domain optical coherence tomography reveal flow directions at retinal vessel bifurcations. Acta Ophthalmol. 2014;92(5):461-464.

6. Ahmed HJ, Klefter ON, Willerslev A, Munch IC, Larsen M. Embolus characterization in branch retinal artery occlusion by optical coherence tomography. Acta Ophthalmol. 2015;93(1):95-96.

7. World Medical Association. World Medical Association Declaration of Helsinki: ethical principles for medical research involving human subjects. JAMA. 2013;310(20):2191-2194 doi:10.1001/jama.2013.281053.

8. Wolf S, Arend O, Reim M. Measurement of retinal hemodynamics with scanning laser ophthalmoscopy: reference values and variation. Surv Ophthalmol. 1994;38(suppl):S95-S100.

9. Wolf S, Jung F, Kiesewetter H, Körber N, Reim M. Video fluorescein angiography: method and clinical application. Graefes Arch Clin Exp Ophthalmol. 1989:227(2):145-151.

10. Arend O, Harris A, Martin BJ, Holin M, Wolf S. Retinal blood velocities during carbogen breathing using scanning laser ophthalmoscopy. Acto Ophthalmol (Copenh). 1994;72(3):332-336.

11. Harris A, Arend O, Wolf S, Cantor LB, Martin BJ. $\mathrm{CO} 2$ dependence of retinal arterial and capillary blood velocity. Acta Ophthalmol Scand. 1995;73(5): 421-424.

12. Rechtman E, Harris A, Kumar R, et al. An update on retinal circulation assessment technologies. Curr Eye Res. 2003;27(6):329-343.

13. Riva CE, Geiser M, Petrig BL; Beijing 100193 , PR China Ocular Blood Flow Research Association. Ocular blood flow assessment using continuous laser Doppler flowmetry. Acta Ophthalmol. 2010;88 (6):622-629.

14. Stalmans I, Vandewalle $E$, Anderson DR, et al. Use of colour Doppler imaging in ocular blood flow research. Acta Ophthalmol. 2011;89(8):e609-e630.
15. Sugiyama T, Araie M, Riva CE, Schmetterer L, Orgul S. Use of laser speckle flowgraphy in ocular blood flow research. Acta Ophthalmol. 2010;88(7): 723-729.

16. Klefter ON, Lauritsen AO, Larsen M. Retinal hemodynamic oxygen reactivity assessed by perfusion velocity, blood oximetry and vessel diameter measurements. Acta Ophthalmol. 2015;93 (3):232-241

17. Jia Y, Bailey ST, Hwang TS, et al. Quantitative optical coherence tomography angiography of vascular abnormalities in the living human eye. Proc Natl Acad Sci U S A. 2015;112(18):E2395-E2402.

18. Meisner JK, Sumer S, Murrell KP, Higgins TJ, Price RJ. Laser speckle flowmetry method for measuring spatial and temporal hemodynamic alterations throughout large microvascular networks. Microcirculation. 2012;19(7):619-631.

19. Zhong Z, Petrig BL, Qi X, Burns SA. In vivo measurement of erythrocyte velocity and retinal blood flow using adaptive optics scanning laser ophthalmoscopy. Opt Express. 2008;16(17): 12746-12756.

20. Garhofer G, Werkmeister R, Dragostinoff N, Schmetterer L. Retinal blood flow in healthy young subjects. Invest Ophthalmol Vis Sci. 2012;53(2): 698-703.

21. Pournaras CJ, Riva CE. Retinal blood flow evaluation. Ophthalmologica. 2013;229(2):61-74

22. Aschinger GC, Schmetterer L, Doblhoff-Dier V, et al. Blood flow velocity vector field reconstruction from dual-beam bidirectional Doppler OCT measurements in retinal veins. Biomed Opt Express. 2015;6(5):1599-1615.

23. Chu YK, Hong YT, Byeon $\mathrm{SH}$, Kwon OW. In vivo detection of acute ischemic damages in retinal arterial occlusion with optical coherence tomography: a "prominent middle limiting membrane sign." Retina. 2013;33(10):2110-2117. 